University of Montana

ScholarWorks at University of Montana

\title{
Light Scattering Studies of Rotational and Vibrational Relaxations of Acetonitrile in Carbon Tetrachloride
}

\author{
Scott L. Whittenburg \\ University of Montana - Missoula, scott.whittenburg@umontana.edu \\ C. H. Wang \\ University of Utah
}

Follow this and additional works at: https://scholarworks.umt.edu/chem_pubs

Part of the Biochemistry Commons, Chemistry Commons, and the Physics Commons Let us know how access to this document benefits you.

\section{Recommended Citation}

Whittenburg, Scott L. and Wang, C. H., "Light Scattering Studies of Rotational and Vibrational Relaxations of Acetonitrile in Carbon Tetrachloride" (1977). Chemistry and Biochemistry Faculty Publications. 68. https://scholarworks.umt.edu/chem_pubs/68

This Article is brought to you for free and open access by the Chemistry and Biochemistry at ScholarWorks at University of Montana. It has been accepted for inclusion in Chemistry and Biochemistry Faculty Publications by an authorized administrator of ScholarWorks at University of Montana. For more information, please contact scholarworks@mso.umt.edu. 


\title{
Light scattering studies of rotational and vibrational relaxations of acetonitrile in carbon tetrachloride
}

\author{
S. L. Whittenburg and C. H. Wang* \\ Department of Chemistry, University of Utah, Salt Lake City. Utah 84112 \\ (Received 3 June 1976)
}

\begin{abstract}
The rotational and vibrational relaxation times of acetonitrile-carbon tetrachloride solutions were investigated as a function of concentration, viscosity, and temperature using depolarized Rayleigh and Raman scattering. Using a Fabry-Perot interferometer and single frequency laser source, we have shown that reliable results for the single particle orientational correlation times $\left(\tau_{\mathrm{s}}\right)$ for $\mathrm{CH}_{3} \mathrm{CN}$ can be obtained by carrying out a concentration dependent depolarized Rayleigh scattering study. Raman scattering was shown to yield inconsistent results for $\tau_{\mathrm{s}}$ in $\mathrm{CH}_{3} \mathrm{CN}$. At constant viscosity, it was found that the Rayleigh scattering relaxation time $\left(\tau_{\text {Ray }}\right)$ of $\mathrm{CH}_{3} \mathrm{CN}$ in $\mathrm{CCl}_{4}$ does not change with $\mathrm{CH}_{3} \mathrm{CN}$ concentration, indicating that the orientational pair correlation factor of liquid $\mathrm{CH}_{3} \mathrm{CN}$ is close to unity. This result suggests that the dynamic pair correlation in $\mathrm{CH}_{3} \mathrm{CN}$ is just as important as the static pair correlation. The experimental data were also compared with the predictions of the hydrodynamic stick and slip models for a rotational diffusion. The $\mathrm{CH}_{3} \mathrm{CN}$ data were found to be close to the prediction of the slip model. The isotropic relaxation time $\left(\tau_{\text {iso }}\right)$ of the $\mathrm{C} \equiv \mathrm{N}$ stretching mode was also studied as a function of concentration and viscosity using Raman spectroscopy. This viscosity dependence of $\tau_{\text {iso }}$ also decreases with decreasing number density of $\mathrm{CH}_{3} \mathrm{CN}$, suggesting that pair correlations are also important in the Raman scattering of $\mathrm{CH}_{3} \mathrm{CN}$.
\end{abstract}

\section{INTRODUCTION}

Light scattering techniques using a Fabry-Perot interferometer and a double-grating monochrometer are useful in studying rotational and vibrational relaxation processes of small molecules in the liquid state. The low frequency $\left(0.1-300 \mathrm{~cm}^{-1}\right)$ light scattering spectrum of a liquid composed of small optically anisotropic molecules exhibits depolarized components in the region of the laser exciting frequency, in addition to a much more intense polarized component which exhibits the Rayleigh-Brillouin spectra in the $0.1 \mathrm{~cm}^{-1}$ region followed by a monotonically decreasing spectral background extending to several hundred wave numbers. The most characteristic feature of the depolarized spectral component is a sharp central peak having a spectral width of the order of $0.5 \mathrm{~cm}^{-1}$ superimposed upon a broad background also extending, in many cases, to several hundred wave numbers. Although the physical nature of the broad background is rather complex, the sharp central component is now understood to be due to rotational diffusion motion, recognizing the possibility that the rotational motion may couple to the overdamped shear modes in liquids. ${ }^{1}$ Thus, in order to obtain unambiguous information about the rotational diffusion of molecules in the liquid state, it is necessary to resolve the sharp central component. Fabry-Perot interferometry provides an ideal method for this purpose.

In recent years Raman scattering has also been used for the study of molecular rotations in the liquid state. The width of a Raman spectral line arising from internal vibrations of small molecules in a liquid is determined by both the vibrational and rotational relaxation processes. Under certain approximations, information about molecular reorientations can be obtained from Raman scattering, and the method has been used successfully in the studies of several molecular liquids. However, the validity of those approximations have recently been questioned, ${ }^{2,3}$ and unless independent experiments are also carried out for its verification, Raman scattering, when applied to obtain information about the rotational motion, has to be used with caution.

On the other hand, no assumptions are needed when Raman scattering is used to obtain information about the vibrational relaxation. Recent experiments have shown that each vibrational band in a molecule has its unique vibrational relaxation behavior, ${ }^{3,4}$ in contrast to the rotational motion which imposes the same effect for all vibrational bands of a given symmetry. Moreover, the vibrational relaxation time of a totally symmetric mode shows an opposite temperature and viscosity dependence to that of the rotational relaxation time.

It is thus of interest to demonstrate the different types of information that one can extract from a molecular liquid using both depolarized Rayleigh and Raman scattering experiments. We selected liquid acetonitrile for this study because a wealth of data for $\mathrm{CH}_{3} \mathrm{CN}$ obtained using other techniques is available in the literature for comparison. Detailed Raman spectral line shape analyses ${ }^{5-7}$ and temperature dependent depolarized Rayleigh ${ }^{8}$ scattering data of pure liquid $\mathrm{CH}_{3} \mathrm{CN}$ have also been reported.

In this paper we have carried out temperature, concentration, and viscosity dependent studies of $\mathrm{CH}_{3} \mathrm{CN}$ in $\mathrm{CCl}_{4}$ and pointed out that the interpretation of the previous light scattering results is subject to question. We have also compared the Raman and Rayleigh scattering results. The isotropic relaxation time of the $\nu_{2}$ mode has also been measured as a function of viscosity and concentration. The result indicates the presence of pair correlation in Raman scattering, which has been neglected previously.

\section{EXPERIMENTAL}

Dust-free acetonitrile in carbon tetrachloride samples were prepared from spectroquality grade liquids and were directly filtered through $0.22 \mu \mathrm{m}$ millipore filters into high quality rectangular glass sample cells for the light scattering experiments. For temperature variation the rectangular glass cell with sample was 


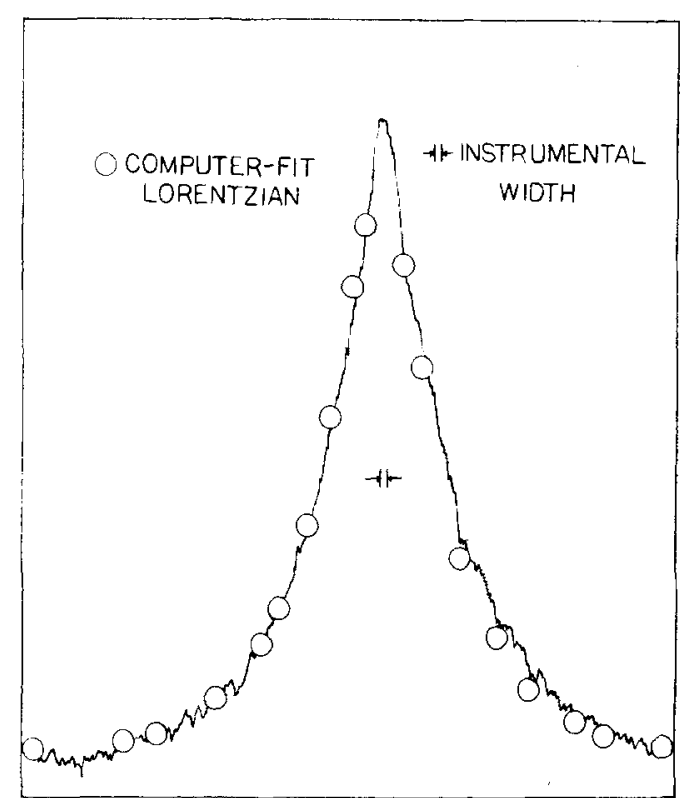

FIG. 1. The measured and calculated depolarized Rayleigh spectra of pure $\mathrm{CH}_{3} \mathrm{CN}$ liquid at room temperature $(294 \mathrm{~K})$.

placed in an aluminum block which was wrapped with heating tape. The accuracy of the temperature at the sample is within $\pm 1 \mathrm{~K}$. The viscosities of all of the samples at various temperatures were measured using a Cannon-Ubbelhode viscometer thermostated in a constant temperature bath. To ascertain the concentration values, an index of refraction vs concentration curve was first constructed from solutions of known concentrations; the index of refraction of the solution used in the experiment was then measured before and after each phase of the experiment, and from the index of refraction values we obtained accurate concentration values for the solutions used in the light scattering experiment.

The depolarized Rayleigh scattering spectra were taken with an optical system consisting of a single frequency Spectra-Physics 165 argon ion laser operated at $4880 \AA$, a Burleigh piezoelectrically scanned FabryPerot interferometer, a cooled photomultiplier tube, a picoammeter, and an $x-y$ recorder. The free spectral range of the interferometer was $860 \mathrm{GHz}$, corresponding to a Fabry-Perot plate separation of $0.17 \mathrm{~mm}$. The free spectral range was checked with the spectral doublet from a sodium lamp and with the depolarized Rayleigh spectrum of toluene. The finesse of the optical system was close to 100 . The detailed description of the experimental setup has been given elsewhere. ${ }^{9}$ The same samples were employed for the Raman measurements. The light source was a Coherent Radiation Laboratories model 54 argon ion laser operating at $4880 \AA$. The scattered light in the $90^{\circ}$ scattering geometry was passed through a polarizer to separate the polarized and depolarized components. The chosen component was then passed through a quartz scrambler and frequency analyzed with a Spex grating double monochromator. The intensity was measured with a cooled ITT FW-130 photomultiplier with the resulting signal displayed on a strip-chart recorder.

The instrumental width was found as a function of slitwidth using the laser line. All Raman spectra were taken at slitwidths of $50-100-50 \mu$, corresponding to an instrumental HWHH of 0.65 wave numbers. The spectra were corrected for this instrumental width.

\section{RESULTS AND DISCUSSION}

\section{A. The single particle correlation time}

We have obtained depolarized Rayleigh scattering data of pure $\mathrm{CH}_{3} \mathrm{CN}$ and solutions of $\mathrm{CH}_{3} \mathrm{CN}$ in $\mathrm{CCl}_{4}$. All experimental Rayleigh spectra were corrected for the instrumental width and the overlap of adjacent orders. A nonlinear least squares program was used to fit a Lorentzian function to the experimental spectra. A typical experimental spectrum and computer fit are shown in Fig. 1, along with the instrumental width. Rayleigh relaxation times $\tau_{\mathrm{Ray}}$ were obtained from the half-width at half-height (HWHH in $\mathrm{Hz}$ ) of the Lorentzian spectra according to

$$
\tau_{\mathrm{R}_{\mathrm{ay}}}=\left(2 \pi \times \mathrm{HWHH}^{-1}\right. \text {. }
$$

The $\tau_{\mathrm{R}_{2 y}}$ values for the pure $\mathrm{CH}_{3} \mathrm{CN}$ and $\mathrm{CH}_{3} \mathrm{CN}-\mathrm{CCl}_{4}$ solutions at various temperatures, along with the $\eta / T$ values ( $\eta$ is the viscosity of the sample, $T$ is absolute temperature) are given in Table I. The relaxation times were reproducible to better than $10 \%$. An experimental accuracy greater than this value is difficult to accomplish in a Fabry-Perot interferometric experiment. Our $\tau_{\mathrm{R}_{a y}}$ value for pure $\mathrm{CH}_{3} \mathrm{CN}$ at room temperature $\left(297^{\circ} \mathrm{K}\right)$ is $1.8 \pm 0.2 \mathrm{psec}$, which is in agreement with the value reported by Patterson and Griffiths. ${ }^{8}$ No published solution data values for $\tau_{\mathbf{R}_{\mathbf{R}}}$ exist, to our knowledge.

$\mathrm{CH}_{3} \mathrm{CN}$ is a symmetric top molecule of $\mathrm{C}_{3 V}$ symmetry. Reorientation about the $C_{3}$ symmetry axis does not modulate the components of the molecular polarizability tensor and thus does not induce depolarized Rayleigh scattering. However, reorientations about the axis

TABLE I. Rayleigh relaxation times for acetonitrile.

\begin{tabular}{llll}
\hline \hline mole $\%$ & $\eta / T(\mathrm{cp} / \mathrm{K})$ & $\tau_{\text {Ray }}$ & $\begin{array}{l}\text { Other } \\
\text { studies }\end{array}$ \\
\hline $100 \%$ & 0.00129 & 1.8 & $1.8^{\mathrm{a}}$ \\
& 0.00122 & 1.8 & \\
& 0.00115 & 1.6 & \\
& 0.00109 & $1.5(5)$ & \\
$81 \%$ & 0.00157 & $2.3(5)$ & \\
& 0.00149 & 2.1 & \\
& 0.00140 & 2.0 & \\
& 0.00133 & 1.9 & \\
$62 \%$ & 0.00190 & 2.8 & \\
& 0.00185 & 2.7 & \\
& 0.00171 & 2.6 & \\
& 0.00165 & 2.4 & \\
$40 \%$ & 0.00237 & 3.5 & \\
& 0.00221 & 3.2 & \\
& 0.00209 & 2.9 & \\
\hline \hline
\end{tabular}

${ }^{2} 298 \mathrm{~K}$, Ref. 8. 


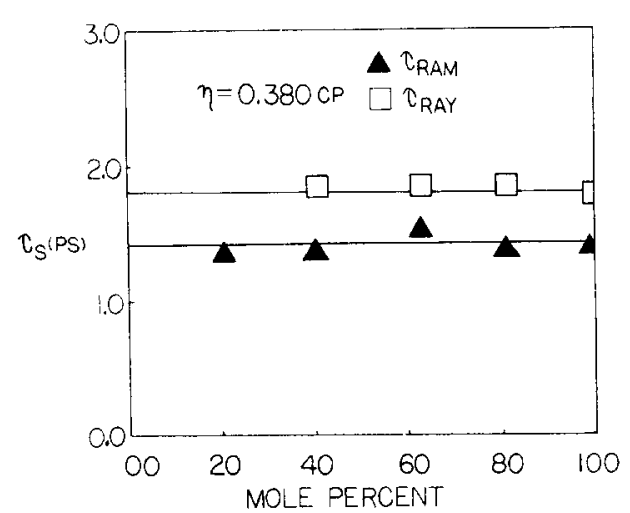

FIG. 2. $\tau_{\text {Ray }}$ and $\tau_{\mathrm{R}}$ of $\mathrm{CH}_{3} \mathrm{CN}$ in $\mathrm{CCl}_{4}$ as a function of concentration at a constant viscosity.

perpendicular to the $C_{3}$ axis induce depolarized Rayleigh scattering. Thus, assuming the rotational diffusion model for reorientations, one expects that the depolarized Rayleigh spectra of $\mathrm{CH}_{3} \mathrm{CN}$ in the pure liquid or in $\mathrm{CCl}_{4}$ solutions should consist of one Lorentzian, as was observed (Fig. 1)。 The quantity $\tau_{\mathrm{R}_{\text {ay }}}$ as defined in Eq. (1) is thus a measure of the average rate of reorientation of the $C_{3}$ axis, as the $\mathrm{CCl}_{4}$ solvent has only negligible depolarized Rayleigh scattering. However, owing to the fact that Rayleigh scattering is a coherent process, the spectral line shape obtained in the depolarized Rayleigh scattering depends on both the single particle reorientational motion and the correlated reorientations of an interacting molecular pair. ${ }^{9}$ Therefore, $\tau_{\mathbf{R}_{\mathbf{a}} \mathbf{y}}$ measured in the pure $\mathrm{CH}_{3} \mathrm{CN}$ liquid does not necessarily represent the single particle reorientation time.

It is possible to obtain the single particle reorientation time from the Rayleigh scattering experiment. According to Keyes and Kivelson, ${ }^{10} \tau_{\mathrm{Ray}}$ and $\tau_{s}$ are related by the equation

$$
\tau_{\mathrm{R} \text { ay }}=\tau_{\mathrm{s}}\left(\frac{1+f n}{1+g n}\right),
$$

where $n$ is the number density; $f$ and $g$ measure the strength of static and dynamic pair correlations, respectively. Thus, in the limit $n \rightarrow 0, \tau_{\mathrm{R} \text { ay }}$ becomes $\tau_{\mathrm{s}}$ of the pure liquid, provided that the viscosity of the solutions is maintained to be equal to that of the pure liquid.

To be sure, the above approximation requires two assumptions: (1) The single particle correlation time $\tau_{\mathrm{s}}$ depends only on solution viscosity and not the specific nature of the solvent used. (2) The pair correlation factors $f$ and $g$ are not dependent on viscosity. Recent experimental work has shown that in the absence of strong interactions (such as hydrogen bonding, dimerization, etc.), these assumptions are justified. 9,11

We have carried out the measurements of $\tau_{\mathrm{Ray}}$ at various solute concentrations. The results are also shown in Table I. Also shown in Fig. 2 is a plot of $\tau_{\text {Ray }}$ (for a fixed $\eta / T$, corresponding to the room temperature value) as a function of $\mathrm{CH}_{3} \mathrm{CN}$ concentration. $\tau_{\mathrm{R}_{2 y}}$ data presented in Fig. 2 are the viscosity weighted values, due to the fact that $\mathrm{CH}_{3} \mathrm{CN}$ has a low viscosity at room temperature $\left(\eta=0.345 \mathrm{cp}\right.$ at $\left.298^{\circ} \mathrm{K}\right)$ and it is difficult to prepare an optically isotropic solvent having this viscosity value which can mix homogeneously with $\mathrm{CH}_{3} \mathrm{CN}$. However, several recent experimental studies have shown that at a fixed temperature $\tau_{\text {Ray }}$ is linearly proportional to viscosity, and it is thus proper to scale the measured $\tau_{\mathrm{R} \text { ay }}$ values at other viscosities to the value corresponding to that of pure $\mathrm{CH}_{3} \mathrm{CN}$.

As one sees in Fig. 2, $\tau_{\mathrm{Ray}}$ for $\mathrm{CH}_{3} \mathrm{CN}$ does not change with the concentration of $\mathrm{CH}_{3} \mathrm{CN}$. The extrapolated value of $\tau_{\mathrm{R}_{2 y}}$ at infinite dilution is $1.8 \pm 0.2$ psec. On the basis of the above discussion, this value should correspond to the single particle correlation time, $\tau_{a}$, of $\mathrm{CH}_{3} \mathrm{CN}$ at room temperature.

It is also possible to determine $\tau_{s}$ by Raman scattering. Raman scattering is generally assumed to be an incoherent process, and the Raman spectral line shape is determined by the single particle relaxation processes. Under favorable conditions, the polarized and the depolarized components of the Raman scattered light from a totally symmetric vibrational mode of a symmetric top molecule can be used to obtain the orientational correlation function. The single particle orientational correlation time is the area under the correlation function. As mentioned above, at a given temperature $\tau_{\mathrm{s}}$ is proportional only to the viscosity of the solution. ${ }^{11}$ Therefore, at a fixed viscosity, as the composition of the solution is varied, one does not expect $\tau_{s}$ as determined by the Raman band shape analysis to vary with the $\mathrm{CH}_{3} \mathrm{CN}$ concentration, and the value of $\tau_{s}$ determined by Raman scattering should be equal to the Rayleigh scattering value extrapolated to infinite dilution. ${ }^{9}$ Thus, concentration dependent Raman scattering experiments can be used to provide a test of the validity of the approximations used in the Raman line shape analysis. Moreover, independent experiments using Raman and Rayleigh scattering can yield an internal consistency check of the $\tau_{\mathrm{a}}$ value as determined by the two different scattering experiments.

We have measured both the polarized and depolarized components of the $\nu_{2}$ vibration of $\mathrm{CH}_{3} \mathrm{CN}$ in $\mathrm{CCl}_{4}$ as a function of temperature and a function of $\mathrm{CH}_{3} \mathrm{CN}$ concentration, using the same sample employed for the Rayleigh scattering experiment. The $\nu_{2}$ vibration corresponds mainly to the symmetric stretch of the $C \equiv N$ bond. Representative polarized and depolarized spectra are given in Fig. 3. The depolarized spectrum is on a greatly expanded scale. All of the totally symmetric vibrations in $\mathrm{CH}_{3} \mathrm{CN}$ are inadvertently handicapped by some secondary structure, thus preventing a straightforward line shape analysis. However, the $\nu_{2}$ band is handicapped the least of the totally symmetric bands, despite an asymmetry appearing at the low frequency sepertal region. Although the asymmetric spectrum can be resolved graphically and the components analyzed to yield the orientational correlation function (from which the zero frequency correlation time can be obtained), we have found that a simpler procedure can be used for the spectral analysis with similar accuracy. ${ }^{12}$ This consists of measuring the half-width at half-height of the Raman spectral intensity using only the high fre- 


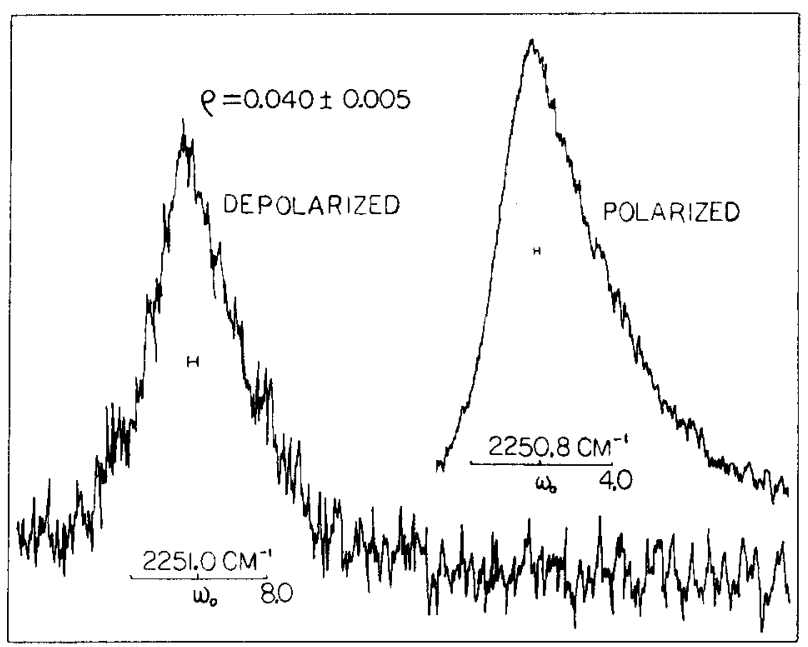

FIG. 3. Representative polarized and depolarized Raman spectra of the $\nu_{2}$ vibration of $\mathrm{CH}_{3} \mathrm{CN}$. Note the asymmetry of the low frequency side. $\rho$ is the depolarization ratio determined by integrating the intensities. The depolarization ratio determined using the peak intensity is considerably smaller.

quency portion of the spectral components. The Raman reorientational $\left(\tau_{R}\right)$ and nonreorientational (or isotropic) oorrelation times [the nonreorientational correlation time $\left(\tau_{\text {iso }}\right)$ will be discussed in the next section] are obtained using the equations

$$
\begin{aligned}
& \tau_{\text {iso }}^{-1}=\tau_{\text {pol }}^{-1}-\tau_{\text {ins }}^{-1}, \\
& \tau_{\mathrm{R}}^{-1}=\tau_{\text {ded }}^{-1}-\tau_{\text {pol }}^{-1},
\end{aligned}
$$

where $\tau_{\text {ins }}$ is the correction due to the instrumental width. $\tau_{\text {dep }}$ and $\tau_{\text {pol }}$ are related to the depolarized and polarized spectral widths, respectively. The experimental polarized spectra were corrected for the additional depolarized component. The expression relating $\tau$ to the spectral half-width at half-height (HWHH) is given by $\tau=(2 \pi c \tilde{\nu})$, where $c$ is the speed of light and $\bar{\nu}$ is the HWHH in $\mathrm{cm}^{-1}$. Experimental values of $\tau_{\mathrm{R}}$ and $\tau_{\text {1so }}$ at $294 \pm 0.1 \mathrm{~K}$ are given in Table II as a function of viscosity along with published values for pure $\mathrm{CH}_{3} \mathrm{CN}$. Although there are no solution data available for comparison, our result for pure $\mathrm{CH}_{3} \mathrm{CN}$ at room temperature agrees well with the value reported by Jones, Anderson, and Pecor $\mathrm{a}^{7}$ and by Bartoli and Litovitz. ${ }^{5}$ However, our value (1.4 psec) differs from Griffiths' reported $\tau_{R}$ value $(0.9 \mathrm{psec})$. This discrepancy lies in the fact that the $\nu_{1}$ band was used by Griffiths to obtain $\tau_{\mathrm{R}}$, and our value (as well as that of Jones et al. ${ }^{7}$ and Bartoli and Litovitz ${ }^{5}$ ) was obtained using the $\nu_{2}$ band. Griffiths favored the $\nu_{1}$ band and has criticized the usage of the $\nu_{2}$ and $\nu_{4}$ bands for the band shape analysis in $\mathrm{CH}_{3} \mathrm{CN}$ owing to the presence of some secondary structure in these bands. ${ }^{6}$ However, the situation is not better when the $\nu_{1}$ band is used for analysis, owing to the fact that in the depolarized spectrum, the scattering spectral density from the $\nu_{5}$ vibration (the $\mathrm{C}-\mathrm{H}$ bending mode) overlaps greatly with $\nu_{1}$. This can introtroduce a greater error in the determination of $\tau_{R}$ using the $\nu_{1}$ band.

\section{B. The pair correlation}

In Table II, one notes that $\tau_{\mathrm{R}}$ of the $\mathrm{CH}_{3} \mathrm{CN}-\mathrm{CCl}_{4}$ solution decreases with increasing $\mathrm{CH}_{3} \mathrm{CN}$ concentration. This decrease is due to the viscosity decrease of the solution because $\mathrm{CCl}_{4}$ has a greater viscosity than $\mathrm{CH}_{3} \mathrm{CN}$. If the $\tau_{\mathrm{R}}$ values of the $\mathrm{CH}_{3} \mathrm{CN}-\mathrm{CCl}_{4}$ solutions are normalized to the values corresponding to the viscosity of pure $\mathrm{CH}_{3} \mathrm{CN}$, then $\tau_{\mathrm{R}}$ assumes a more or less constant value (1.4 psec) over the concentration range stucied (see Fig. 2).

It is interesting to note that $\tau_{\mathrm{R}}$ determined for pure $\mathrm{CH}_{3} \mathrm{CN}$ using Raman scattering is slightly smaller than the $\tau_{s}$ value obtained by Rayleigh scattering $(1.4$ psec obtained using Raman scattering vs 1.8 psec obtained using Rayleigh scattering). Although the difference is less than the combined experimental uncertainty of Raman and Rayleigh scattering, we feel that the single particle reorientation time of $\mathrm{CH}_{3} \mathrm{CN}$ at room temperature is close to $1.8 \mathrm{psec}$, as the analysis of the Rayleigh data to obtain $\tau_{s}$ does not require the various assumptions used in Raman scattering.

The result of the concentration-dependent Rayleigh scattering study also allows us to discuss the importance of pair orientational correlations of $\mathrm{CH}_{3} \mathrm{CN}$ in the liquid state. Using the assumption that $\tau_{s}$ is equal to $\tau_{\mathrm{R}}$ obtained from the Raman spectral line shape analysis of the $\nu_{1}$ band, Patterson and Griffiths have previously reported the pair orientational correlation factor $(1+f n) /(1+g n)$ in pure $\mathrm{CH}_{3} \mathrm{CN}$ using the combined Rayleigh and Raman results. ${ }^{8}$ However, as pointed out above, the values of $\tau_{\mathrm{R}}$ in $\mathrm{CH}_{3} \mathrm{CN}$ is not unique. For the $\nu_{1}$ band, $\tau_{R}$ is $0.9 \mathrm{psec}$, and it is $1.4 \mathrm{psec}$ for both $\nu_{2}$ and $\nu_{4}$ bands. Therefore, the accuracy of the paircorrelation factor reported in Ref. 8 is subject to question.

It should be emphasized that the method of using Raman scattering to obtain the single particle correlation time need be used with care. This method will give inaccurate results when the isotropic (or nonreorientational) relaxation mechanism is also important in affecting the line shape of a Raman band. For $\mathrm{CH}_{3} \mathrm{CN}$ the isotropic relaxation mechanism is not unimportant (Sec. IV). As mentioned above, the $\tau_{\mathrm{Ray}}$ at infinite dilution equals $\tau_{\mathrm{s}^{\circ}}$ This $\tau_{\mathrm{s}}$ value is $1.8 \mathrm{psec}$ instead of the 0.9 psec value used in Ref。9. Moreover, our $\tau_{\text {Ray }}$ data for

TABLE II. Rotational and vibrational Raman relaxation times of acetonitrile.

\begin{tabular}{lllll}
\hline \hline & & $\begin{array}{l}\text { Other } \\
\text { studies }\end{array}$ & $\tau_{\mathrm{R} \text { (psec) }}$ & $\begin{array}{l}\text { Other } \\
\text { studies }\end{array}$ \\
\hline 0.380 & 3.2 & $3.3^{\mathrm{d}} 3.6^{\mathrm{b}}$ & 1.4 & $1.4,^{\mathrm{b}} 1.5^{\mathrm{c}, \mathrm{d}}$ \\
0.461 & 3.1 & & 1.7 & \\
0.560 & $3.0(5)$ & & 2.3 & \\
0.698 & 2.9 & 2.6 & \\
0.820 & $2.8(5)$ & & 2.9 & \\
\hline \hline
\end{tabular}

${ }^{a}$ All measurements made at $294 \pm 0.5 \mathrm{~K}$.

b $308.7 \mathrm{~K}$, Ref. 7 .

c $_{296}$ K, Ref. 5.

$\mathrm{d}_{298 \mathrm{~K}, \text { Ref. } 6 .}$ 


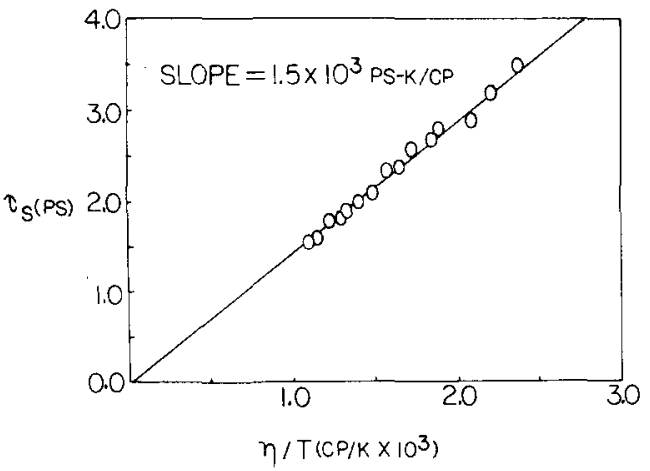

Fig. 4. The plot of $\tau_{\mathrm{s}}$ as a function of $\eta / T$ for $\mathrm{CH}_{3} \mathrm{CN}$. The $\tau_{s}$ values were obtained from Rayleigh scattering data.

the $\mathrm{CH}_{3} \mathrm{CN}$ solution, when adjusted to correspond to $\tau_{\mathrm{R}_{\text {ay }}}$ for the pure liquid, show only an insignificant concentration dependence. In view of Eq. (2), this result suggests that the pair correlation factor $(1+f n) /(1+g n)$ is close to unity, implying that the static and the dynamic correlation factors are of the same size (i. e., $f=g$ ).

In principle, it is possible to determine $f$ by measuring the integrated intensity of the depolarized Rayleigh scattering spectrum. Malmberg and Lippincott ${ }^{13}$ and Coumou et al. ${ }^{14}$ have done such measurements for several liquids composed of optically anisotropic molecules in optically isotropic solvents. For the $\mathrm{CH}_{3} \mathrm{CN}-\mathrm{CCl}_{4}$ system, the relative depolarized Rayleigh scattering intensity increases from about 0.3 to 0.72 as the mole fraction of $\mathrm{CH}_{3} \mathrm{CN}$ increases from 0 to $1 .{ }^{13}$ These results indicate that static pair correlations are present in $\mathrm{CH}_{3} \mathrm{CN}$. It also shows that the local field fluctuation of the isotropic solvent (such as $\mathrm{CCl}_{4}$ ) contributes significantly to the integrated Rayleigh intensity. At present, there is no reliable method available to allow the separation of the effects of solute and solvent molecules upon the internal field. As a result, we cannot estimate the static correlation factor of $\mathrm{CH}_{3} \mathrm{CN}$ from their intensity measurement.

Previously it was generally believed that the dynamic correlation factor $g n$ was small compared to the static factor $f n$. If this is also true in $\mathrm{CH}_{3} \mathrm{CN}$, then on the basis of Eq. (2) $f n$ is also small, since the experimental result requires that $f=g$. It would be worthwhile to find some independent experiment to verify the present result.

\section{The Stokes-Einstein relation}

With the availability of temperature and viscosity dependent reorientation times, we can discuss the applicability of the various Stokes-Einstein models for the calculation of $\tau_{\mathrm{s}}$ in $\mathrm{CH}_{3} \mathrm{CN}$. The Stokes-Einstein equation relates the rotational diffusion coefficient $D_{R}$ to the friction coefficient $\zeta$ by $D_{R}=k T / \zeta$, where $\zeta=8 \pi \eta a^{3}$ for a spherical particle of radius $a$ immersed in a continuum fluid of viscosity $\eta$ assuming "stick" boundary conditions. Perrin has extended the calculation to include frictional coefficients for prolate and oblate ellipsoids. ${ }^{15}$ The ellipsoidal formulas are simply the spherical for- mulas multiplied by functions of the axial ratio.

For symmetric-top molecules and in the rotational diffusion approximation, $\tau_{s}$ is related to $D_{R}$ (the rotational diffusion coefficient of the symmetry axis) by

$$
\tau_{\mathrm{s}}=\left(6 D_{R, 1}\right)^{-1}=C(\eta / T)
$$

where the constant $C$ is determined by the axial ratio and other molecular constants. The plot of $\tau_{\mathrm{s}} \mathrm{vs} \eta / T$ for $\mathrm{CH}_{3} \mathrm{CN}$ is shown in Fig. 4. A linear least-squares fit of the data to the equation

$$
\tau_{\mathrm{s}}=\tau_{0}+\mathrm{C} / \eta
$$

gives $C=(1.5 \pm 0.05) \times 10^{3} \mathrm{psec} \cdot \mathrm{K} / \mathrm{cp}$ and $\tau_{0}=(0.0 \pm 0.05)$ psec.

This value of $\tau_{0}$ is consistent with the zero intercept predicted by the Stokes-Einstein relation. Some previous depolarized Rayleigh scattering studies have found a nonvanishing $\tau_{0}$. The nonvanishing $\tau_{0}$ value was interpreted as being associated with the classical free rotation of the molecule. However, free-rotor reorientation occurring at long times (in the time scale of the depolarized Rayleigh scattering) is inconsistent with the derivation of the Stokes-Einstein relation. Nevertheless, for all the molecules which we have studied, we have found that, within the experimental error, $\tau_{0}$ is equal to zero.

The experimental value of the slope $C$ is compared with the predictions of the various Stokes-Einstein models in Table III. The slope calculated from Eq. (5) and ${ }^{15}$

$$
D_{R, \perp}^{-1}=\frac{8 \pi a b^{2}}{k}(\eta / T)
$$

is a factor of 9 too great. In Eq. (7), $a$ and $b$ are the long and short molecular axis of $\mathrm{CH}_{3} \mathrm{CN}$ which were determined from space-filled models and the formula provided by Edwards. ${ }^{16}$

There is no obvious reason for the Stokes-Einstein theory to be applicable to small molecules in the pure liquid state or in a solvent consisting of molecules of a similar size, as the theory uses stick boundary conditions which assume that the fluid molecules adjacent to the rotating particle sticks to the particle and rotates with it. However, experimentally it has been shown that a linear dependence of $\tau_{\mathrm{s}}$ on $\eta / T$ is quite general and is independent of the assumed stick boundary conditions. Recent theoretical calculations of the friction coefficient $\zeta$ have been carried out for ellipsoids with slip boundary conditions. ${ }^{17,18}$ The calculated slope $C$

TABLE III. Comparison to Stokes-Einstein relations. ${ }^{2}$

\begin{tabular}{ll}
\hline \hline Experimental slope & $1.5 \pm 0.05 \mathrm{psec}-\mathrm{K} / \mathrm{cp}$ \\
Experimental intercept & $0.0 \pm 0.05 \mathrm{psec}$ \\
$\begin{array}{l}\text { Slope calculated from Stokes-E instein } \\
\text { using slip boundary conditions }\end{array}$ & $2 \pm 1 \times 10^{3} \mathrm{psec}-\mathrm{K} / \mathrm{cp}$ \\
$\begin{array}{l}\text { Slope calculated from Stokes-Einstein } \\
\text { using stick boundary conditions }\end{array}$ & $9 \pm 2 \times 10^{3} \mathrm{psec}-\mathrm{K} / \mathrm{cp}$ \\
\hline
\end{tabular}

${ }^{\mathbf{a}}$ Modeling acetonitrile as an ellipsoid. 
using slip boundary conditions is also shown in Table III; the value is in a closer agreement with the experimental value. The large uncertainty in the calculated value is due to the difficulty in modeling $\mathrm{CH}_{3} \mathrm{CN}$ as an ellipsoid. Nevertheless, the result shows that, like other fluids of small molecules, when the $\mathrm{CH}_{3} \mathrm{CN}$ molecule rotates in the liquid state it slips over the neighboring molecules freely. The viscosity dependence of $\tau_{s}$ is thus due to the fact that thermal energy must be spent to create sufficient free volume for the molecular rotation to occur. The slip boundary condition is more appropriate to describe the rotation of small molecules in the liquid state.

\section{The nonreorientational relaxation}

We have also studied the isotropic (or nonreorientational) relaxation effect on the line shape of the $\nu_{2}$ Raman band. Although Raman scattering cannot provide an unambiguous correlation time of the $\mathrm{CH}_{3} \mathrm{CN}$ molecule in the liquid state, there is no corresponding difficulty in obtaining information about the nonreorientational process. The depolarization ratio of the $\mathrm{C} \equiv \mathrm{N}$ stretch band is small (about 0.04). Thus, the linewidth of the polarized spectral component is determined almost exclusively by the isotropic relaxation process.

Using Eq. (3), we have determined the isotropic relaxation time $\tau_{1 s_{0}}$ as a function of viscosity and concentration (Table II). The $\tau_{\text {sso }}$ value for the $\nu_{2}$ band for pure $\mathrm{CH}_{3} \mathrm{CN}$ liquid agrees with the $3.3 \pm 0.3 \mathrm{psec}$ value reported in the literature ${ }^{6}$ (within the experimental uncertainty).

The mechanism of vibrational (isotropic) relaxation in typical nonassociated liquids has been considered as due to binary collisions. ${ }^{19}$ The physical basis for use of this model is the assumption that major contribution to vibrational relaxation comes from a very few collisions with exceptionally high energies. Using this assumption, Litovitz ${ }^{20}$ has developed a model which relates the rate of energy transfer to the product of a probability of energy transfer per hard binary collision $P$ times the collision frequency $Z$, which is equal to $\tau_{B C}^{-1}$, where $\tau_{B C}$ is the time between collisions. Campbell, Fisher, and Jonas ${ }^{21}$ have interpreted $\tau_{1 \mathrm{so}}$ of $\mathrm{CH}_{3} \mathrm{I}$ using the binary collision model and found that in order to explain the $\tau_{1 \mathrm{so}}$ result at constant density, it is necessary to assume that the collision frequency decreases with increasing temperature, or the value of $\tau_{B C}$ increases with increasing temperature. ${ }^{21}$

In the dilution experiment as done here, the temperature is fixed, and the concentration is varied. Changing the concentration amounts to changing viscosity of the solution. It is interesting to note that the value of $\tau_{180}$ of $\mathrm{CH}_{3} \mathrm{CN}$ decreases as the viscosity of the solution increases. This viscosity dependence is opposite to that of $\tau_{s}$, and it corresponds to the line narrowing effect of the isotropic part of the polarized Raman spectrum upon increasing temperature already demonstrated in several simple molecular liquids. ${ }^{22}$ The present $\mathrm{CH}_{3}{ }^{\prime} \mathrm{CN}$ experiment involves changing the sample composition and keeping the temperature fixed. However, the line narrowing phenomena is consistent with the temperature dependent results, owing to the fact that increasing the sample temperature decreases its viscosity.

We can discuss the viscosity dependence of $\tau_{\text {iso }}$ by considering another experimental quantity, $\tau_{J}$, the angular momentum correlation time. The angular correlation time $\tau_{J}$ is related to the time it takes for the molecule to lose memory of its initial angular momentum and, as such, in the binary collision model, it is proportional to the time between collisions, $\tau_{B C}$. If one assumes a rotational diffusion model for molecular reorientation, then the Hubbard relation ${ }^{23}$ applies. The Hubbard relation is

$$
\tau_{\mathrm{s}} \tau_{J}=I / 6 k T \text {. }
$$

Substituting $\mathrm{Eq}$. (5) for $\tau_{s}$ we obtain

$$
\tau_{J}=\left(\frac{I}{6 k C}\right) \frac{1}{\eta} \text {. }
$$

Equation (9) shows that $\tau_{J}$ should decrease with increasing viscosity, and if the binary collision model is adopted, we then expect $\tau_{1 \text { so }}$ to increase with decreasing $v$ iscosity, in contradiction to the present experimental result.

The unusual feature of the isotropic Raman scattering spectrum in $\mathrm{CH}_{3} \mathrm{CN}$ is necessarily linked to the vibrational relaxation induced by the $\mathrm{CH}_{3} \mathrm{CN}-\mathrm{CH}_{3} \mathrm{CN}$ and $\mathrm{CH}_{3} \mathrm{CN}-\mathrm{CCl}_{4}$ interactions. These interactions provide pathways for the exchange of energy among the neighboring oscillators as well as the dissipation of the vibrational energy of an oscillator to the nonvibrational degrees of freedom (translations and reorientations of molecules). For the vibrational energy dissipation mechanism to be effective, the fluctuation of the nonvibrational degrees of freedom must be fast enough to pick up the oscillator energy. The frequency of the $\mathrm{C} \equiv \mathrm{N}$ stretch is about $6 \times 10^{13} \mathrm{~Hz}$. Thus, in order to dissipate this vibrational energy, high energy inelastic collisions must occur. However, collisional events at this frequency range are known to have a very small probability. Therefore, this energy dissipation mechanism is not likely to affect the isotropic relaxation process.

The other mechanism involving the exchange of oscillator energy is believed to be much more important in affecting the $\tau_{\text {iso }}$ value. Although resonance excitation energy transfer is an energy conserving process, it involves a phase change of the vibrational wave function of the interacting pair. Because of molecular motions the phase change is a function of time. This is the homogeneous dephasing mechanism which can significantly affect the spectral linewidth, and hence $\tau_{180^{\circ}}$ This homogeneous dephasing mechanism is well known in NMR, but its importance has not been emphasized in Raman scattering spectroscopy.

For the $\mathrm{CH}_{3} \mathrm{CN}$ system, the intermolecular dipoledipole interaction is strong. In fact, the $\mathrm{x}$-ray diffraction measurements of liquid $\mathrm{CH}_{3} \mathrm{CN}$ have shown the existence of molecular clusters involving four molecules of the first coordination sphere and a central molecule. ${ }^{24}$ 


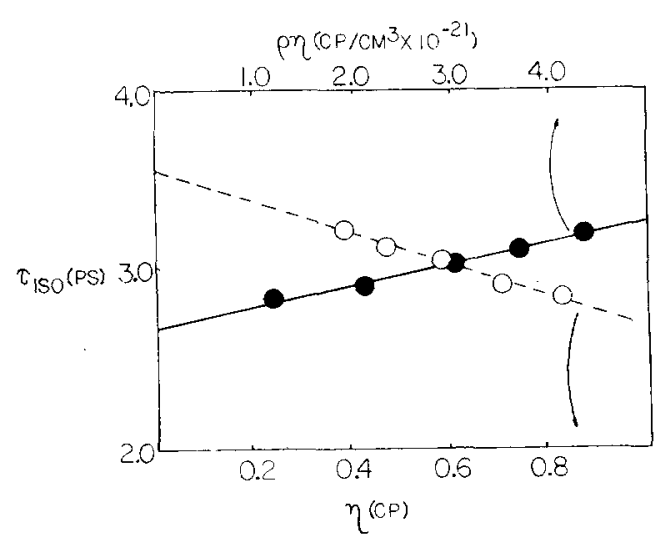

Fig. 5. The isotropic relaxation times $\left(\tau_{\text {iso }}\right)$ measured at room temperature are plotted as a function of $\eta$ and $\rho \eta$.

The coupling is believed to take place via a dipoledipole interaction as the charge distribution of the $C \equiv N$ bond is highly polarized. Of course, the dipole-dipole interaction must be modulated by the molecular vibration in order for it to become an isotropic relaxation mechanism. That is, the vibrational coupling takes place via the interaction of the transition dipoles.

The experimental results obtained for $\mathrm{CH}_{3} \mathrm{CN}$ are consistent with the resonance energy transfer mechanisms due to the (transition) dipole-dipole interaction. A line narrowing phenomenon due to this mechanism is expected, since the molecular motion tends to make the energy exchange process less effective, and hence result in a larger isotropic relaxation time. This is what we observe experimentally and is also borne out in a recent theoretical calculation by $\mathrm{Wang}_{0}{ }^{25}$

It is often assumed that Raman scattering is an incoherent process and therefore only the single particle motion is probed. In the presence of intermolecular coupling, the phases of the vibrational wavefunctions of the interacting pair are no longer independent, and the assumption of incoherence is thus not justified. There is experimental evidence which indicates that this assumption is not applicable in liquid $\mathrm{CH}_{3} \mathrm{CN}$ 。

We have measured $\tau_{\text {soo }}$ as a function of number density of $\mathrm{CH}_{3} \mathrm{CN}$ in $\mathrm{CCl}_{4}$ at room temperature. As is shown in Fig. 5, $\tau_{180}$ decreases with decreasing $\mathrm{CH}_{3} \mathrm{CN}$ concentration. In fact, this decrease outweighs the increase due to the viscosity increase upon dilution (see Fig. 5 for the plots of $\tau_{\text {iso }}$ vs $\eta$ and $\rho \eta$, where $\rho$ is the number density of $\mathrm{CH}_{3} \mathrm{CN}$ ). This experimental result indicates that the spectral line of the $\mathrm{C} \equiv \mathrm{N}$ stretch is narrowed by the $\mathrm{CH}_{3} \mathrm{CN}-\mathrm{CH}_{3} \mathrm{CN}$ interaction.

If one assumes that $\tau_{\text {iso }}$ is the single particle vibrational correlation time of the $\mathrm{C} \equiv \mathrm{N}$ mode, it is a simple matter to show that isotropic relaxation $r$ ate $\left(\tau_{\text {iso }}^{-1}\right)$ is proportional to $\rho$, the number density of $\mathrm{CH}_{3} \mathrm{CN}$ molecules. Thus, the decrease of $\tau_{\text {iso }}$ with decreasing $\rho$ is not expected for the single particle time. The pair correlation due to the dipole-dipole interaction probably plays an important role in affecting the unusual density dependence.

Other experiments have shown the importance of vibrational pair correlation on the $\mathrm{C} \equiv \mathrm{N}$ vibration. $\mathrm{Re}-$ cent ir matrix isolation work $\mathrm{k}^{26}$ indicates that the $\mathrm{C}-\mathrm{N}$ linkage of one $\mathrm{CH}_{3} \mathrm{CN}$ molecule is coupled to the neighboring one via the structure

$$
\begin{aligned}
\mathrm{CH}_{3}-\mathrm{C} & \equiv \mathrm{N} \\
\dot{\mathrm{N}} & \equiv \dot{\mathrm{C}}-\mathrm{CH}_{3} .
\end{aligned}
$$

This intermolecular coupling may be related to the high boiling point of the $\mathrm{CH}_{3} \mathrm{CN}$ liquid (b. p. $82^{\circ} \mathrm{C}$, in contrast to $\mathrm{CH}_{3} \mathrm{C} \equiv \mathrm{C}-\mathrm{H}$, which is about $-23^{\circ} \mathrm{C}$; both have the same molecular weight.)

It is possible to incorporate the pair-correlation effect in formulating a theory for Raman scattering. Using the Mori type of linear response theory, ${ }^{27}$ Wang has shown that in Raman scattering, the pair-correlation affects the spectral line shape and the peak position, but not the intensity. ${ }^{15}$ This situation is quite different from the Rayleigh scattering case in which the presence of pair-correlation affects both the integrated intensity and linewidth. This difference is due to the fact that in Raman scattering only the dynamic pair correlation is present, while in Rayleigh scattering both static and dynamic pair correlations are present.

We know very little about the dynamic pair correlation at present. The investigation of $\tau_{\mathrm{R}}$ and $\tau_{\text {iso }}$ as a function of concentration should yield information on the effect of the dynamic pair correlation of a Raman spectral line in a molecular liquid.

\section{SUMMARY AND CONCLUSION}

Using depolarized Rayleigh and Raman scattering techniques, we have studied the rotational and vibrational relaxation times of acetonitrile as a function of concentration, viscosity, and temperature. We have shown that, while Raman scattering may yield inconsistent results for the single particle orientational correlation time for $\mathrm{CH}_{3} \mathrm{CN}$, reliable results can be obtained using Rayleigh scattering by carrying out concentration dependent studies using a Fabry-Perot interferometer. We have found that at constant viscosity the Rayleigh scattering relaxation times $\left(\tau_{\mathrm{R}_{\text {ay }}}\right)$ of $\mathrm{CH}_{3} \mathrm{CN}$ in $\mathrm{CCl}_{4}$ do not change with $\mathrm{CH}_{3} \mathrm{CN}$ concentration, indicating that the dynamic orientational pair correlation in $\mathrm{CH}_{3} \mathrm{CN}$ is important. Other experimental results have indicated that the static orientational pair correlation in $\mathrm{CH}_{3} \mathrm{CN}$ is present. We have found that the Raman relaxation time $\left(\tau_{r}\right)$ extracted from the $\nu_{2}$ band does not agree with the single particle reorientation time $\tau_{s}$ determined by Rayleigh scattering although the difference

TABLE IV. Scaled ${ }^{2}$ relaxation times for acetonitrile.

\begin{tabular}{ccccc}
\hline \hline mole $\%$ & $\tau_{\text {RAM }}^{\text {ROT }}$ & \multicolumn{1}{c}{$\tau_{\text {Ray }}$} & \\
\hline 100 & 1.4 & 0.3 & 1.8 & 0.2 \\
81 & 1.4 & 1.9 & \\
62 & 1.6 & 1.9 & \\
40 & 1.4 & 1.9 & \\
20 & 1.3 & $\cdots$ & \\
\hline
\end{tabular}

aScaled to an $\eta$ value of $0.380 \mathrm{cp}, T=294 \mathrm{~K}$. 
is small. We have also shown that results from previous light scattering studies on pure liquid $\mathrm{CH}_{3} \mathrm{CN}$ are not consistent with the present concentration dependent Rayleigh scattering data. Concentration dependent studies are necessary to yield information on the pair correlations.

We have obtained single particle orientational correlation times as a function of viscosity and temperature. The experimental data have been compared with those predicted by the hydrodynamic stick and slip models. Our results are close to the prediction using the slip model.

The isotropic relaxation time $\left(\tau_{1 \mathrm{so}}\right)$ of the $\mathrm{C} \equiv \mathrm{N}$ stretching mode has also been studied as a function of concentration and viscosity. $\tau_{\text {tso }}$ is found to decrease with increasing viscosity. This viscosity dependence is in a direction opposite to that of the orientation correlation time $\tau_{\mathrm{s}}$, but is consistent with the line narrowing effect observed in the temperature dependent study of the isotropic parts of the polarized Raman scattering spectra of simple molecular liquids. The number density of $\mathrm{CH}_{3} \mathrm{CN}$ has a significant effect on $\tau_{1 \mathrm{so}}$. We have found that the isotropic relaxation time $\tau_{\text {sso }}$ decreases with decreasing number density, suggesting that the pair correlation is also important in the Raman scattering of $\mathrm{CH}_{3} \mathrm{CN}$.

\section{ACKNOWLEDGMENTS}

We acknowledge the support of the Donors of the Petroleum Research Fund, administered by the American Chemical Society, for support of this research. We also thank Dr. Dane R. Jones for repeating parts of the experiments.

\footnotetext{
*Alfred P. Sloan Foundation Fellow.

${ }^{1}$ The couplings of the rotational motion to the shear modes was observed originally by V. S. Starunov, E. V. Tiganov, and I. L. Fabelinskii, JETP Lett. 5, 260 (1967).

${ }^{2}$ C. H. Wang, D. R. Jones, and D. H. Christensen, J. Chem.
}

Phys. 64, 2820 (1976).

${ }^{3}$ D. H. Christensen and O. F. Nielsen, J. Chem. Phys. (to be published).

${ }^{4}$ W. G. Rothchild, G. J. Rosasco, and R. C. Levingston, J. Chem. Phys. 62, 1253 (1975).

${ }^{5}$ F. J. Bartoli and T. A. Litovitz, J. Chem. Phys. 56, 413 (1972).

${ }^{6}$ J. E. Griffiths, J. Chem. Phys. 59, 751 (1973).

${ }^{7}$ D. R. Jones, H. C. Anderson, and R. Pecora, Chem. Phys. 9, 339 (1975).

${ }^{8}$ G. D. Patterson and J. Griffiths, J. Chem. Phys. 63, 2406 (1975).

${ }^{9}$ C. K. Cheung, D. R. Jones, and C. H. Wang, J. Chem. Phys. 64, 3576 (1976).

${ }^{10}$ T. Keyes and D. Kivelson, J. Chem. Phys. 56, 1057 (1972).

${ }^{11}$ G. R. Alms, D. R. Bauer, J. I. Brauman, and R. Pecora, J. Chem. Phys. 58, 5570 (1973); ibid. 59, 5310 (1973).

${ }^{12}$ The zero-frequency correlation times were also obtained from computing the correlation functions using the symmetric part of the spectrum. The results were found to be the same as the simple analysis as discussed herein.

${ }^{13} \mathrm{M}$. S. Malmberg and E. R. Lippincott, J. Colloid Interface Sci. 27, 591 (1968).

${ }^{14}$ D. J. Coumou, J. Hijmans, and E. L. Mackor, Trans. Faraday Soc. 60, 2244 (1964).

${ }^{15}$ F. Perrin, J. Phys. Radium 5, 33 (1934); ibid. 34, 399 (1965).

${ }^{16}$ J. T. Edward, Jr. Chem. Educ. 47, 261 (1970).

${ }^{17} \mathrm{C}$. Hu and R. Zwanzig, J. Chem. Phys. 60, 4354 (1.974).

${ }^{18}$ G. K. Youngren and A. Acrivos, J. Chem. Phys, 63, 3846 (1975).

${ }^{19} \mathrm{~K}$. Herzfeld and T. A. Litovitz, Absorption and Dispersion of Ultrasonic Waves (Academic, New York, 1959).

${ }^{20}$ T. A. Litovitz, J. Chem. Phys. 26, 469 (1957; W. M. Madigosky and T. A. Litovitz, ibid. 34, 489 (1961).

${ }^{21}$ J. H. Campbell, J. F. Fisher, and J. Jonas, J. Chem. Phys. 61, 346 (1974).

${ }^{22}$ R. B. Wright, M. Schwartz, and C. H. Wang, J. Chem. Phys. 58, 5125 (1973); ibid. 59, 5259 (1973).

${ }^{23}$ P. S. Hubbard, Phys. Rev. 131, 1155 (1963).

${ }^{24}$ A. Kratochwill, J. U. Weidner, and H. Zimmerman, Ber. Bunsenges. Phys. Chem. 34, 407 (1973).

${ }^{25} \mathrm{C}$. H. Wang, J. Chem. Phys. (submitted).

${ }^{26}$ T. B. Freedman and E. R. Nixon, Spectrochim. Acta Part A 28, 1375 (1972).

${ }^{27}$ H. Mori, Prog. Theor. Phys. 33, 423 (1965); ibid. 34, 399 (1965). 